\title{
MODELING THE RADIAL FORGING OF HOLLOW INGOTS IN MULTI-DIE UNITS
}

\author{
A. N. Shubina, N. A. Babailov* \\ Institute of Engineering Science, Ural Branch of the Russian Academy of Sciences, \\ 34, Komsomolskaya st., Ekaterinburg, Russian Federation \\ *Corresponding author. E-mail: babailov@imach.uran.ru \\ Address for correspondence: 34, ul. Komsomolskaya, 620049, Ekaterinburg, Russian Federation \\ Tel.: +7 (343) 37535 96; fax: +7 (343) 3745330
}

The paper reports on the results of modeling the swaging of hollow preforms in a multi-die forging unit. The deformation of a continuously cast hollow preform produced by castingdeformation modules to be rolled, e.g. in multi-roll mills, is studied. To estimate the adequacy of the theoretical model of forming in the deformation of the hollow preform, we studied the deformation of the preform on model materials, particularly, with the use of lead. Critical strain is determined, i. e. the strain at the moment of hollow closing to obtain a solid billet. The main parameters affecting critical reduction are the initial geometry of the hollow ingot and the relative feed of the ingot into the dies. The ingots are deformed on a special experimental 8-die unit providing a closed deformation zone in the whole interval when the dies close up. The device is designed to model swaging of preforms made of a model material, up to $45 \mathrm{~mm}$ in diameter. The deformation is performed by radial swaging with displacement. The processing of the experimental data by regression analysis offers the values of the coefficients of approximating equations for the obtained experimental data. The experimental data are compared with the calculation results for a mathematical model of hollow ingot deformation, based on Kolmogorov's variational method. The use of the Fisher criterion demonstrates a good agreement of the measurement results with the theoretical data obtained from mathematical models. reduction.

Keywords: continuously cast ingot, multi-die unit, swaging, modeling material, critical

DOI: $10.17804 / 2410-9908.2017 .4 .006-015$

1. Korshunov E.A., Kobyakov N.S., Kostrov V.P., Melnikov E.V. Continuous casting and combined metal processing with a repeated upward billet discharge from the mold. Izvestiya VUZov. Chernaya metallurgiya, 1987, no. 10, pp. 140-141. (In Russian).

2. Burkin S.P., Korshunov E.A., Shakhpazov E.Kh., Smirnov V.K., Babailov N.A., Loginov Yu.N. Minimization of consumable power during manufacturing the steel rolled products in the casting-and-deforming modules. Stal, 1996, no. 6, pp. 29-33. (In Russian).

3. Bourkine S.P., Loginov Y.N., Shimov V.V., Babailov N.A. Energy analysis of a through-put radial forging machine. Journal of Materials Processing Technology, 1999, vol. 86, iss. 1-3, pp. 291-299.

4. Bourkin S.P., Korshunov E.A., Loginov Y.N., Mironov G.V. A method of production of steel and the unit for its implementation. RU Patent 2133650, 1999. (In Russian).

5. Bourkin S.P. Mironov G.V., Korshunov E.A., Loginov Y.N. Metal complex to produce. RU Patent 2106930, 1998. (In Russian).

6. Burkin S.P., Korshunov E.A., Shimov V.V., Babailov N.A., Andryukova E.A. Razrabotka mashin i tekhnologiy metallurgii pri innovatsionnom riske [Development of Machinery and Technologies of Metallurgy with Innovation Risk, S.P. Burkin, ed.: Textbook]. Ekaterinburg, UrFU Publ, 2010, 498 p. (In Russian).

7. Volodin A.M., Lazorkin V.A., Petrov N.P., Nikitin V.V. Four-die forging apparatus. $R U$ Patent 2314175, 2008, Bul. no. 1. (In Russian). 
8. Volodin A.M., Lazorkin V.A. On the advantage of bar forging on a hydraulic forging presses in four-die forging devices. Kuznechno-Shtampovochnoe Proizvodstvo. OMD, 2009, no. 11, pp. 17-23. (In Russian).

9. V.A. Tyurin, V.A. Lazorkin, I.A. Pospelov, G.P. Flakhovsky, eds. Kovka na radialnoobzhimnykh mashinakh [Forging on Radial-Swaging Machines]. M., Mashinostroenie, 1990, 256 p. (In Russian).

10. Kolmogorov V.L. Mekhanika obrabotki metallov davleniem [Mechanics of Metal Forming]. Ekaterinburg, UGTU-UPI Publ., 2001, 836 p. (In Russian).

11. Burkin S.P., Babailov N.A. Studying the forming of a hollow continuously cast billet. Izvestiya VUZov. Chernaya Metallurgiya, 1997, no. 9, pp. 40-44. (In Russian).

12. Burkin S.P., Babailov N.A. Energy and force parameters of a hollow billet under radial swaging. Kuznechno-Shtampovochnoe Proizvodstvo, 1997, no. 8, pp. 8-10. (In Russian).

13. Babailov N.A., Bourkin S.P. The mathematical model of radial forging of a hollow continuously cast ingot. In: R.J. Furness et al. eds. Proceedings of the ASME Manufacturing Engineering Division, MED, vol. 11, NY, ASME, 2000, pp. 515-522.

14. Babailov N.A., Burkin S.P., Loginov Yu.N. On the determination of strain resistance in radial swaging by a multi-die unit. Izvestiya VUZov. Chernaya metallurgiya, 1998, no. 6, pp. 31-32. (In Russian).

15. Babailov N.A., Loginov Yu.N. Forging press block having a yoke with a rotary drive. RU Patent 2016131011, 2017, Bul. no. 20. (In Russian).

16. Kassandrova O.N., Lebedev V.V. Obrabotka resultatov nabludeniy [Processing of Observation Results]. M., Nauka Publ., 1970, 104 p. (In Russian). 
Подана в журнал: 17.08 .2017

УДК 621.73.012

DOI: $10.17804 / 2410-9908.2017 .4 .006-015$

\title{
МОДЕЛИРОВАНИЕ РАДИАЛЬНОЙ КОВКИ ПОЛОЙ ЗАГОТОВКИ В МНОГОБОЙКОВЫХ БЛОКАХ
}

\author{
А. Н. Шубина, Н. А. Бабайлов* \\ Федеральное государственное бюджетное учреждение науки Институт машиноведения \\ Уральского отделения Российской академии наук, Екатеринбург, Российская Федерация \\ *Ответственный автор. Электронная почта: babailov@imach.uran.ru \\ Адрес для переписки: 620049, Екатеринбург, ул. Комсомольская, 34, Российская Федерация \\ Тел.: +7 (343) 375-35-96; факс: +7 (343) 374-53-30
}

В статье представлены результаты моделирования протяжки полых заготовок в многобойковом ковочном блоке. Исследуется процесс деформации непрерывно-литой полой заготовки, получаемой на литейно-деформационных модулях, с целью дальнейшей прокатки, например, в многовалковых прокатных станах. Для оценки адекватности теоретической модели формоизменения при деформации полой заготовки проведено исследование деформации заготовки на модельных материалах, в частности с использованием свинца. В работе определялась критическая степень деформации - момент заковки полости заготовки (или смыкания стенок заготовки), с целью получении сплошной заготовки. Основные параметры, влияющие на критическое обжатие - начальная геометрия полой заготовки и относительная подача заготовки в бойки блока. Деформация заготовок была осуществлена на специальном 8-бойковом экспериментальном блоке, который обеспечивает замкнутый очаг деформации во всем интервале при смыкании бойков. Это устройство предназначено для моделирования проходной радиальной ковки заготовок из модельного материала с диаметром до 45 мм. При деформации заготовки осуществляется процесс радиального обжатия со сдвигом. При обработке опытных данных с помощью методов регрессионного анализа получены значения коэффициентов аппроксимирующих уравнений для полученных экспериментальных данных. Приведено сравнение опытных данных с расчетными результатами для математической модели деформации полой заготовки на основе вариационного метода В.Л. Колмогорова. Использование критерия Фишера в данной работе показало хорошую сходимость полученных результатов измерений и полученных теоретических данных на основе математических моделей.

Ключевые слова: непрерывно-литая заготовка, многобойковый блок, проходная радиальная ковка, протяжка, заковка полости, модельный материал, критическое обжатие.

\section{1. Введение}

В настоящее время актуальным направлением развития металлургических технологий является использование ресурсосберегающих совмещенных процессов литья и пластической деформации заготовок [1-7], а также создание литейно-деформационных агрегатов (модулей) различного состава и конструкции. Такие технологии и машины позволяют значительно уменьшить расход энергии на вторичный нагрев металла заготовок перед пластической деформацией, а также повысить производительность процессов обработки металлов давлением (например, ковки, прокатки и др.).

В работе рассмотрен вопрос изготовления сплошной заготовки из полой непрерывнолитой заготовки для последующей сортовой прокатки в многовалковых прокатных блоках. В литературе известны конструкции машин и технологические особенности ковки на радиально-обжимных машинах (РОМ) или радиально-ковочных машинах (РКМ) [8-10]. В лите- 
ратуре также рассматривается деформация заготовки при сочленении машин непрерывного литья заготовок с РОМ или РКМ, имеющих в своей конструкции 4 и более бойков.

В работе исследован процесс получения сплошной заготовки методом проходной многобойковой ковки на плоских бойках. Непрерывно-литая полая заготовка получается разливкой вверх [3] с целью получения качественного коркового мелкозернистого металла. В дальнейшем кованая заготовка предназначена для последующей непрерывной прокатки.

Для определения формоизменения полой заготовки ранее получены решения с использованием математической модели, разработанной на основе вариационного метода В.Л. Колмогорова [11]. В работах $[12,13,14]$ на основе минимума полной мощности деформации определено перераспределение металла между радиальным и осевым направлением, изменение толщины стенки полой заготовки (утонение или утолщение) и критическое обжатие, т. е. момент заковки полости заготовки (смыкание стенок заготовки).

\section{2. Материал и методика}

В работе представлены результаты физического моделирования заковки полости непрерывно-литой заготовки из модельного материала. Известно, что свинцовые сплавы являются широко используемым модельным материалом в обработке металлов давлением при моделировании горячей деформации стали и др. материалов. Эксперименты выполнены на полых цилиндрических образцах с концентрическим отверстием. Образцы изготавливались из предварительно прессованной заготовки диаметром 18 мм (из свинца марки С1 по ГОСТ 3778-98 «Свинец. Технические условия»). Длина заготовки после поперечной разрезки прутка составляла от 80 до 100 мм. Для снижения эксцентриситета внутреннее отверстие в заготовке изготовлено сверлением на токарном станке.

Исследованы особенности заковки полости заготовки в зависимости от следующих относительных параметров процесса:

- геометрического параметра сечения полой заготовки $k=D_{0} / d_{0}$, зависящего от начальных размеров заготовки;

- относительной подачи заготовки в бойки блока $l_{0}=L_{0} / L_{5}$, являющейся технологическим параметром процесса ковки (протяжки), где $D_{0}$ и $d_{0}-$ наружный и внутренний диаметры полой заготовки соответственно; $L_{0}$ - подача заготовки в бойки ковочного блока; $L_{5}$ - ширина плоских бойков многобойкового блока.

В работе экспериментально определена относительная деформация в момент заковки полости, или так называемое критическое обжатие, по формуле [15]:

$$
q_{K}=\left(D_{0}-D_{0 K}\right) / D_{0}
$$

где $D_{0 K}$ - диаметр вписанной окружности поперечного сечения образца в момент заковки полости.

Величина подачи заготовки в бойки при ковке (протяжке) ограничивается величиной обжатия заготовки (т. е. вытяжки заготовки вдоль направления деформации) и шириной плоских бойков ковочных агрегатов. При проведении экспериментов ширина бойков лабораторной установки может варьироваться за счет использования сменных накладок бойков.

Деформация полой (трубчатой) заготовки осуществляется до момента смыкания стенок заготовки (трубы) и закрытия полости, схема процесса радиального обжатия полой заготовки приведена на рис. $1 a$.

Экспериментальные исследования выполнены на 8-бойковом ковочном блоке, предназначенным для деформирования заготовок из модельных материалов с максимальным диаметром до 45 мм. Блок был смонтирован на универсальной испытательной машине УИМ-30 (в исследовательской лаборатории кафедры «Обработка металлов давлением» Уральского федерального университета) [15]. 

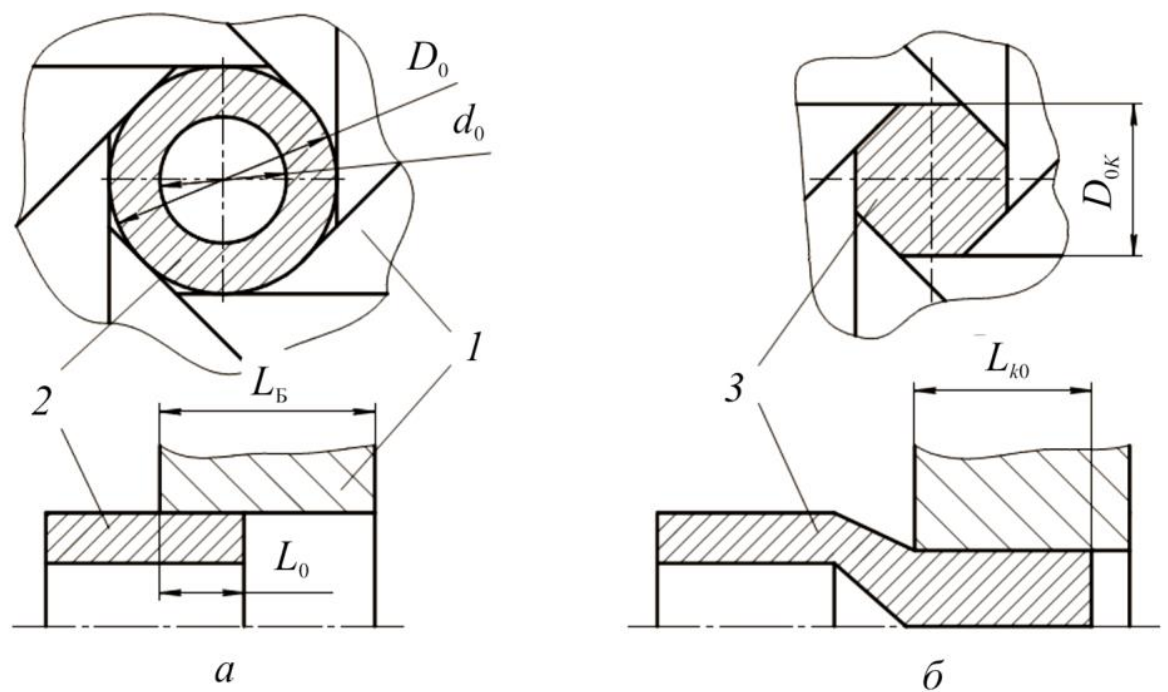

Рис. 1. Схема деформации полой заготовки до деформации $(a)$ и после деформации (б): 1 - бойки; 2 - исходная заготовка; 3 - деформированная заготовка с закованной полостью (стрелкой показано направление подачи заготовки)

Деформация полой заготовки осуществляется до момента смыкания стенок заготовки (трубы) и закрытия полости, схема процесса приведена на рис. 1 б.

Экспериментальные исследования выполнены на 8-бойковом ковочном блоке, предназначенном для деформирования заготовок из модельных материалов с максимальным диаметром до 45 мм. Блок был смонтирован на универсальной испытательной машине УИМ-30 (в исследовательской лаборатории кафедры Обработка металлов давлением Уральского федерального университета) [15].

Многобойковый ковочный блок представлен на рис. 2. Восемь бойков (поз. 1) перемещаются по направляющим 2, закрепленным в обойме 3. Количество бойков может быть 3 , 4, 6 или 8, причем большее их число нежелательно из-за высоких потерь на трение между бойками. Корпус ковочного блока устанавливается на нижнюю траверсу испытательной машины УИМ-30. Обойма 3 закрепляется на колоннах испытательной машины посредством двух специальных зажимов, состоящих из полукольца 4 и корпуса зажима 5. Корпус зажима на обойме 3 закрепляется двумя болтами.

Привод бойков осуществляется при повороте обоймы 3 относительно корпуса ковочного блока 4 за счет приложенного крутящего момента. Механизм перемещения бойков сверху закрыт накладкой, прикрепляемой к бойку 1 тремя винтами.

На рис. 2 представлена 8-бойковая экспериментальная установка для моделирования процессов проходной радиальной ковки (протяжки) заготовок [16]. Установка предназначена для радиального обжатия сплошных и полых свинцовых заготовок с наружным диаметром до 45 мм. Особенностью конструкции ковочного блока является, то, что при деформации заготовки за счет поперечного к оси подачи заготовки смещения бойков осуществляется процесс радиального обжатия со сдвигом.

Величина подачи заготовки в бойки при ковке (протяжке) ограничивается величиной обжатия заготовки (вытяжки заготовки вдоль направления деформации) и шириной бойков ковочных агрегатов. Ширина бойков блока $L_{\mathrm{b}}$ лабораторной установки может варьироваться от 18 до 40 мм за счет использования сменных накладок, которые крепятся к торцевым поверхностям бойков (поз. 1 , рис. 2). 


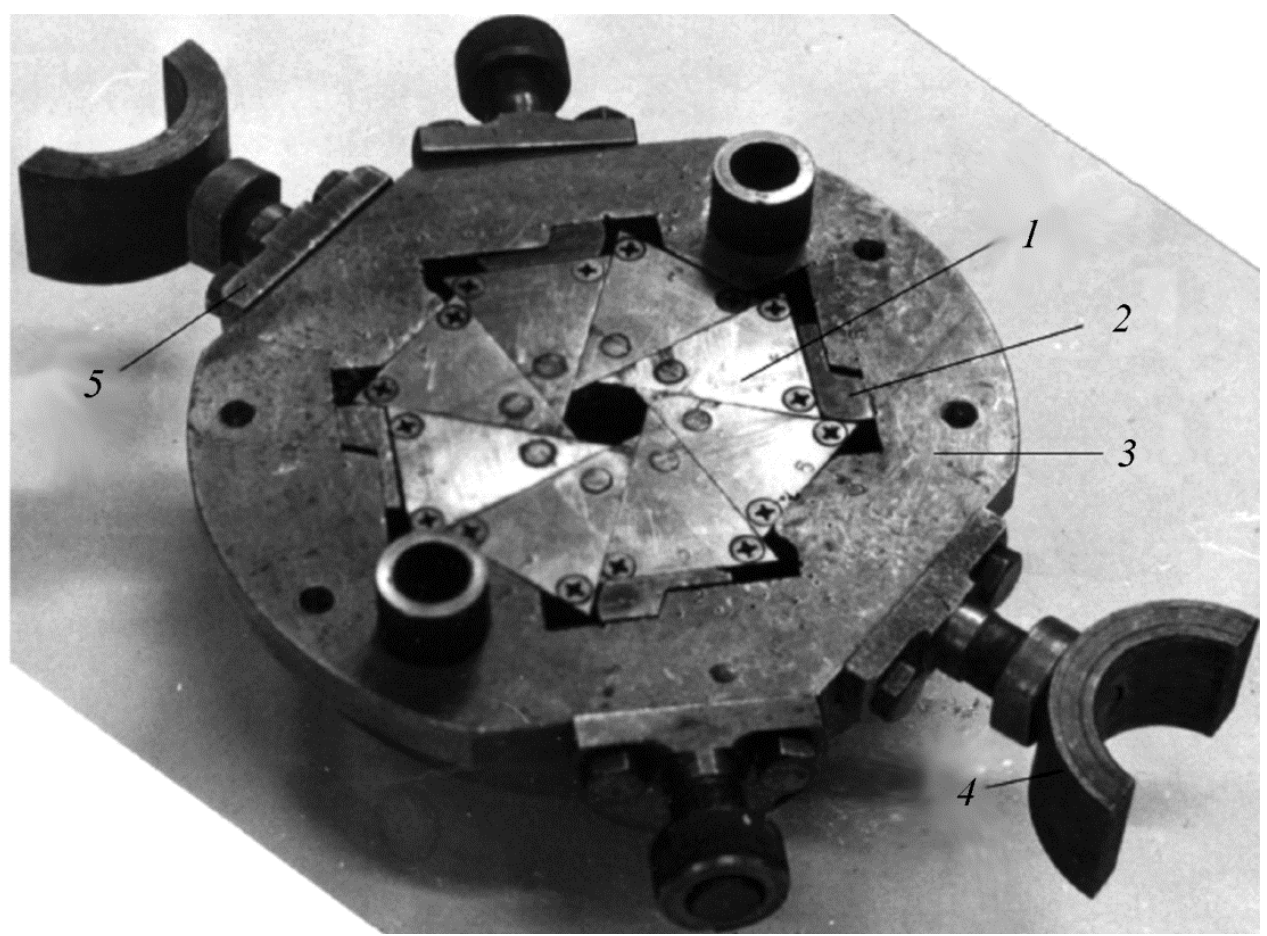

Рис. 2. 8-бойковый блок для моделирования протяжки заготовок:

1 - боек; 2 - направляющая; 3 - обойма; 4 - полукольцо; 5 - корпус зажима

\section{3. Результаты и обсуждение}

Проведены замеры начальных размеров заготовки для выбранных трех типов заготовок с концентрическим отверстием (табл. 1). Измерения выполнены при помощи штангенциркуля ЩЦ 1-125 с ценой наименьшего деления, равной 0,05 мм. Для каждой заготовки выполнено по $n=5$ замеров наружного и внутреннего диаметров заготовок. При малом количестве испытаний $n<30$ использовано распределение Стьюдента [17]. Исключение промахов для всех результатов испытаний выполнено по критерию Пирсона.

Значения геометрического параметра сечения полой заготовки (средние значения и границы доверительного интервала для надежности $P=0,95$ и количестве измерений, равным $n=5)$ представлены в табл. 1.

Таблица 1 - Геометрические размеры исследуемых наборов образцов и погрешности результатов измерения для $P=0,95$ и $n=5$

\begin{tabular}{|c|c|c|c|}
\hline Тип образца & $D_{0}$, мм & $d_{0}$, мм & $k$ \\
\hline 1 & \multirow{3}{*}{$18,01 \pm 0,049$} & $10,01 \pm 0,053$ & $0,556 \pm 0,003$ \\
\cline { 3 - 4 } \cline { 3 - 4 } & & $8,53 \pm 0,050$ & $0,472 \pm 0,003$ \\
\cline { 3 - 4 } & & $7,34 \pm 0,038$ & $0,406 \pm 0,002$ \\
\hline
\end{tabular}

Для построения каждой точки на графиках было выполнено по 5 испытаний образцов. Образцы (для каждого набора параметров $k$ и $l_{0}$ ) были продеформированы в многобойковом блоке за 3 обжатия (или шага нажатия бойков) с соответствующей подачей заготовки в бойки блока.

Деформация образцов осуществлялась до смыкания внутренней поверхности образца. Далее на строгальном станке удалять половину деформированного образца, и выполняли измерение диаметра вписанной окружности поперечного сечения образца в момент заковки полости штангенциркулем ЩЦ 1-125. Выполнено по $n=5$ замеров $D_{0 K}$ на одном образце. 
На рис. 3. представлен деформированный образец с выполненными 3 этапами (шагами) обжатий заготовки после удаления половины заготовки, обозначено место измерения диаметра вписанной окружности поперечного сечения образца в момент заковки полости на 2-м шаге обжатия.

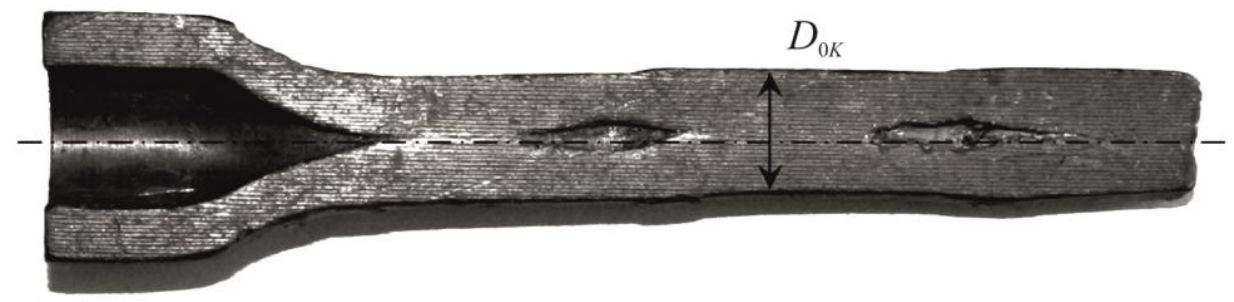

Рис. 3. Деформированный образец

Обработка результатов испытаний выполнена аналогично представленной методике с определением начальных размеров образцов: с использованием распределения Стьюдента, исключение промахов выполнено по критерию Пирсона. Результаты обработки экспериментальных данных - зависимость критического обжатия от относительной подачи заготовки для различных параметров заготовки представлены на рис. 4. На графиках приведены средние значения измеряемой величины, а штрихами - максимальное $(\max )$ и минимальное $(\min )$ значения доверительного интервала.

С использованием метода регрессионного анализа, экспериментальные результаты измерения показателя критического обжатия полой заготовки аппроксимированы следующим уравнением:

$$
y=A \cdot x^{B}
$$

где $A$ и $B$ - коэффициенты уравнения (2), значения которых приведены в табл. 2.

На рис. 4 красная линия - теоретические значения изучаемого показателя, полученного по математической модели, а тонкая черная линия - линия по уравнению (2).

В табл. 2 приведена также максимальная относительная погрешность результатов в серии измерений для различных параметров заготовки $k$.

Таблица 2 - Коэффициенты аппроксимации в модели (2)

\begin{tabular}{|c|c|c|c|c|}
\hline $\begin{array}{c}\text { Параметр сечения } \\
\text { заготовки } k\end{array}$ & $A$ & $B$ & $R^{2}$ & $\begin{array}{c}\text { Максимальная относительная } \\
\text { погрешность измерений, \% }\end{array}$ \\
\hline 0,556 & 0,5788 & $-0,265$ & 0,9838 & 7,3 \\
\hline 0,472 & 0,5365 & $-0,263$ & 0,9975 & 9,7 \\
\hline 0,406 & 0,5071 & $-0,249$ & 0,9901 & 7,2 \\
\hline
\end{tabular}



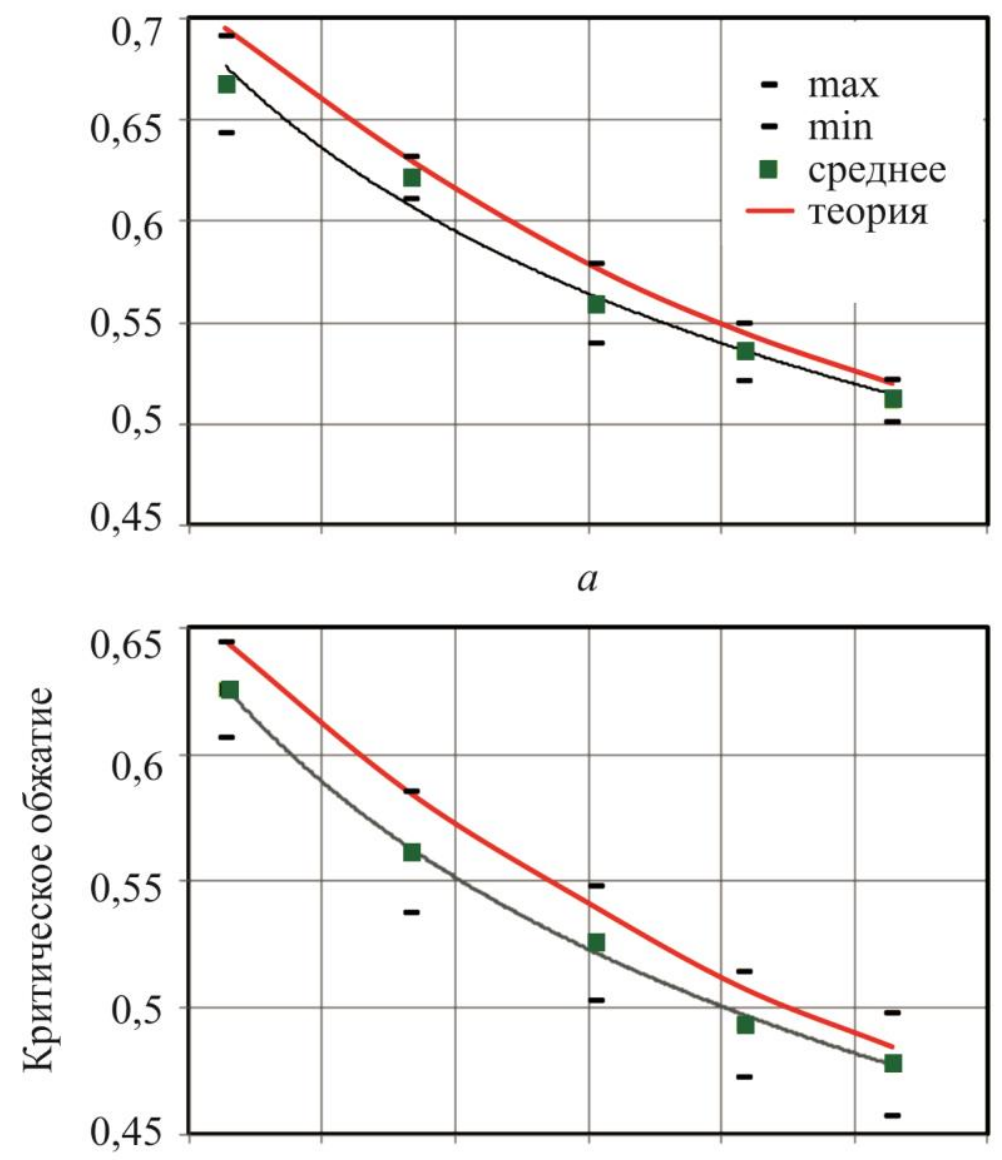

$\sigma$

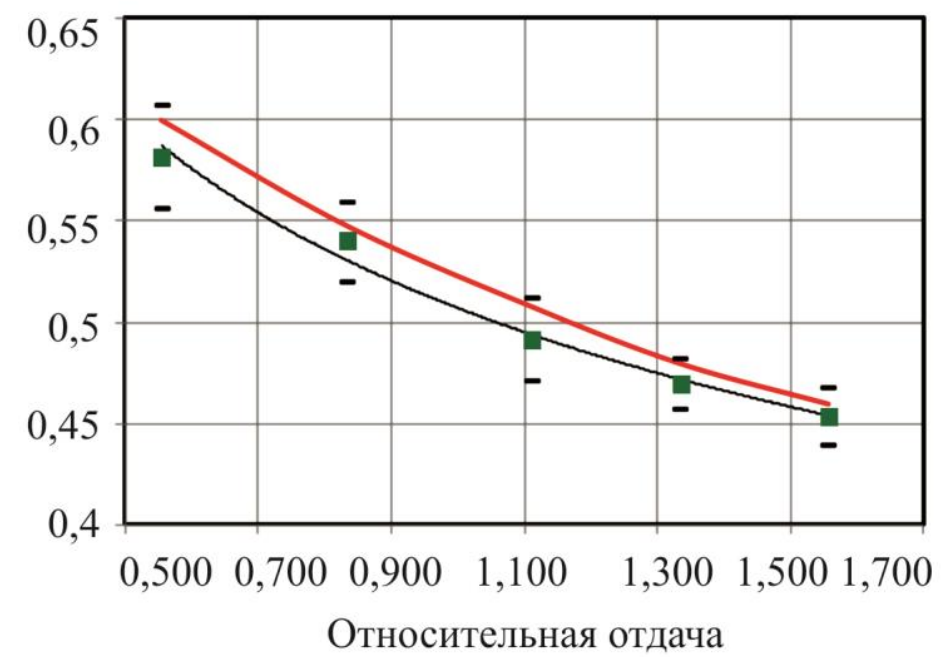

B

Рис. 4 - Зависимость критического обжатия от относительной подачи заготовки для параметра заготовки $k=0,556(a) ; 0,472$ (б) и 0,406 (в)

Полученные результаты показали достаточно хорошую сходимость полученных экспериментальных данных и математической модели процесса, разработанной по методике В.Л. Колмогорова. Следует отметить, что для всех серий испытаний средние значения измерений $q_{K}$ ниже значений, полученных при вычислениях по математической модели процесса. Наиболее близкие опытные результаты к теоретическим данным получены для деформированных заготовок при больших относительных подачах $\left(l_{0}>1\right)$, а также для заготовок с наиболее толстой стенкой $(k=0,406)$. 
Сходимость результатов экспериментов и разработанной математической модели проведена с использованием критерия Фишера. Анализ показал, что полученные значения этого критерия ниже представленных табличных.

Отличие между экспериментальными и теоретическими результатами может возникать вследствие, например, затекания небольшой части металла заготовки в зазоры между движущимися бойками.

\section{4. Заключение}

В работе представлены результаты физического моделирования деформации полой заготовки в процессе проходной ковки (протяжки) и выполнена проверка адекватности математической модели по определению формоизменения полой заготовки Анализ результатов лабораторных исследований показал хорошее соответствие значений критического обжатия, определенного по теоретической модели на основе вариационного принципа В.Л. Колмогорова (минимизация полной мощности деформации) с результатами измерений деформированных образцов.

Результаты показали, что обжатия заготовки более предсказанной в математической модели величины должны обеспечить гарантированную заковку полости заготовки.

Представленные авторами модели позволяют использовать полученные результаты для проектирования технологии проходной ковки (протяжки) в многобойковых блоках с выбором рациональных параметров подач и обжатий, а также ковочное оборудование для осуществления процесса ковки (протяжки).

\section{Благодарность}

Авторы выражают благодарность за помощь в организачии исследований и научное руководство работой С.П. Буркину профессору кафедры Обработка металлов давлением Уральского федерального университета.

\section{Литература}

1. Непрерывная разливка и совмещенная металлообработка при периодической выдаче заготовок из кристаллизатора вверх / Е. А. Коршунов, Н. С. Кобяков, В. П. Костров, Е. В. Мельников // Известия ВУЗов. Черная металлургия. - 1987. - № 10. - С. 140-141.

2. Минимизация потребляемой мощности при производстве стального проката на литейно-деформационных модулях / С. П. Буркин, Е. А. Коршунов, Е. Х. Шахпазов, В. К. Смирнов, Н. А. Бабайлов, Ю. Н. Логинов // Сталь. - 1996. - № 6. -С. 29-33.

3. Energy analysis of a through-put radial forging machine / S. P. Bourkine, Y. N. Loginov, V. V. Shimov, N. A. Babailov // Journal of Materials Processing Technology. 1999. - Vol. 86, iss. 1-3. - P. 291-299. - DOI: 10.1016/S0924-0136(98)00319-7.

4. Способ производства металлопродукции и агрегат для его осуществления : пат. 2133650 Рос. Федерация / Буркин С. П., Коршунов Е. А., Логинов Ю. Н., Миронов Г. В. № 98115371/02 ; заявл. 04.08.1998, опубл. 27.07.1999.

5. Комплекс для получения металлопродукции : пат. 2106930 Рос. Федерация / Буркин С. П., Миронов Г. В., Коршунов Е. А., Логинов Ю. Н. - № 96106819/02 ; заявл. 08.04.1996; опубл. 20.03.1998.

6. Ресурсо- и энергосбережение в металлургии. Разработка машин и технологий металлургии при инновационном риске : учебник : в двух книгах : книга 1 / С. П. Буркин, Е. А. Коршунов, В. В. Шимов, Н. А. Бабайлов, Е. А. Андрюкова ; под общ. ред. С. П. Буркина. - Екатеринбург : УрФУ, 2010. - 498 с. 
7. Четырехбойковое ковочное устройство : пат. 2314175 Рос. Федерация / Володин А. М., Лазоркин В. А., Петров Н. П., Никитин В. В., ОАО «Тяжпрессмаш». - № 2005139668/02 ; заявл. 19.12.2005 ; опубл. 10.01.2008, Бюл. № 1.

8. Володин А. М., Лазоркин В. А. О достоинствах технологии ковки слитков на гидравлических ковочных прессах в четырехбойковых ковочных устройствах // Кузнечноштамповочное производство. ОМД. - 2009. - № 11. - С. 17-23.

9. Ковка на радиально-обжимных машинах / В. А. Тюрин, В. А. Лазоркин, И. А. Поспелов, Г. П. Флаховский / под общ. ред. В. А.Тюрина. - М. : Машиностроение, 1990. - 256 с.

10. Колмогоров В. Л. Механика обработки металлов давлением. - Екатеринбург : УГТУ-УПИ, 2001. - 836 с.

11. Буркин С. П., Бабайлов Н. А. Исследование формоизменения полой непрерывнолитой заготовки // Известия ВУЗов. Черная металлургия. - 1997. - № 9. - С. 40-44.

12. Буркин С. П., Бабайлов Н. А Энергосиловые параметры при радиальном обжатии полой заготовки // Кузнечно-штамповочное производство. - 1997. - № 8. - С. 8-10.

13. Babailov N. A., Bourkin S. P. The mathematical model of radial forging of a hollow continuously cast ingot // Proceedings of the ASME Manufacturing Engineering Division, MED / ed. by R. J. Furness et al. - NY : ASME, 2000. - Vol. 11. - P. 515-522.

14. Бабайлов Н. А., Буркин С. П., Логинов Ю. Н. Об определении сопротивления деформации при радиальном обжатии многобойковым блоком // Известия высших учебных заведений. Черная металлургия. - 1998. - № 6. - С. 31-32.

15. Ковочный блок пресса, имеющего траверсу с приводом вращения : пат. на полезную модель 172547 U1 / Бабайлов Н. А., Логинов Ю. Н. - № 2016131011 ; заявл. 27.07.2016 ; опубл. 11.07.2017, Бюл. № 20.

16. Кассандрова О. Н., Лебедев В. В. Обработка результатов наблюдений. - М. : Наука, 1970. - 104 c. 